\title{
Educación ambiental e cartografía social: experiencias en una comunidad de Holguín, Culba
}

\author{
Environmental education and social cartography: experiences in a \\ community of Holguín, Cuba
}

\section{Olga Alicia Gallardo Milanés ${ }^{1}$ \\ Lais Martinez Gallardo ${ }^{2}$ \\ Angel Reyes Bermúdez ${ }^{3}$}

\begin{abstract}
Palabras-clave:
Cartografía Social

Comunidad

Educación Ambiental

Escuela
\end{abstract}

\begin{abstract}
Resumen
La cartografía social es una importante herramienta para desarrollar acciones de educación ambiental en la comunidad porque promueve la participación activa y crítica de diversos actores sociales. El objetivo de la investigación fue comprender la contribución de la cartografía social a la educación ambiental comunitaria a partir de presupuestos teóricos y prácticos, para ello se estudió el proceso de educación ambiental desarrollado en la comunidad Oscar Lucero Moya de Holguín- Cuba a través del proyecto Mapa Verde. La investigación se realizó desde una perspectiva cualitativa, analizando la realidad desde la complejidad, se efectuaron entrevistas y cuestionarios que permitieron escuchar los criterios de las personas que participaron en el proceso de educación ambiental comunitaria. El estudio permitió concluir que la cartografía social utilizada en la comunidad Oscar Lucero Moya logró potencializar nuevas relaciones sociales con la naturaleza, en la práctica se fortalecieron lazos de cooperación mediante la articulación de actores y se construyeron nuevos saberes que re-significaron el contexto. Además se asumió una nueva cosmosvisión desde un enfoque de sistema complejo, que favoreció el análisis de los conflictos socio-ambientales develados como problemas.
\end{abstract}

Keywords:

Social mapping

Community

Environmental education

School

\begin{abstract}
Social mapping is an important tool to develop environmental education actions in the community because it promotes the active and critical participation of various social actors. The objective of the research was to understand the contribution of social cartography to community environmental education from theoretical and practical budgets, for this we studied the process of environmental education developed in the Oscar Lucero community of Holguin-Cuba through the Green Map project. The research was conducted from a qualitative perspective, analyzing the reality from the complexity, interviews and surveys were conducted that allowed us to listen to the criteria of the
\end{abstract}

\footnotetext{
1 Universidad de Holguín. oaliciagallardo2013@gmail.com

2 Universidad de Holguín. lmartinezg@uho.edu.cu

${ }^{3}$ Universidad de Holguín. areyes@uho.edu.cu
} 
people who participated in the environmental education process. The study allowed us to conclude that the social mapping used in the Oscar Lucero Moya community managed to potentiate new social relations with nature, in practice cooperation ties were strengthened through the articulation of actors and new knowledge was built that re-signified the context. In addition, a new worldview was assumed from a complex system approach, which favored the analysis of socioenvironmental conflicts revealed as problems.

\section{INTRODUCCIÓN}

En la educación ambiental, a pesar de su preocupación común por el medio ambiente, los diferentes autores proponen diversas maneras de concebir y practicar la acción educativa en este campo (SUAVÉ, 2004). Una de esas maneras de hacer educación ambiental puede ser la cartografía social, la que constituye un espacio de producción de saberes; porque permite identificar problemas, conflictos ambientales y posibilita intercambio entre diversos actores sociales; además admite la construcción colectiva de conocimientos.

La cartografía social ha sido utilizada en el mundo, existen varios desarrollos teóricos del mapeo participativo y experiencias que abordan diversos problemas y conflictos ambientales entre ellas Vélez, Rátiva y Varela (2012), Poggi, (2013); Barragan (2016) entre otros. Se ha trabajado en conflictos por el agua, la tierra, en el mapeamiento de pueblos y comunidades tradicionales de la Pan-Amazonía y la cartografía social pedagógica. Sin embargo no se encontraron estudios que muestren como la cartografía social contribuye con la educación ambiental comunitaria, ese fue uno de los propósitos de la investigación.

En Cuba se trabaja la cartografía social participativa para enfrentar problemas ambientales a través de la gestión ambiental comunitaria. El Centro Félix Varela hace más de 20 años ha logrado consolidar en el país una red de Mapa Verde, que a su vez pertenece a una red global de mapeadores, mediante la cual promueve acciones de educación ambiental. Con una estrategia nacional de trabajo realizan proyectos a través de los cuales sus integrantes promueven procesos de gestión ambiental comunitaria participativa. La unidad básica y funcional es el grupo, en el que voluntariamente participan personas de diferentes grupos etarios.

En la provincia Holguín está bien estructurada la red en la que participan grupos de diversas instituciones, ubicados en varios municipios. En la ciudad de Holguín la coordinación la realiza la Universidad de Holguín, la que trabaja la educación ambiental con escuelas y comunidades. La educación ambiental que se realiza promueve la participación, en el país el proceso de participación solamente llega a complementarse en todos sus momentos en el espacio local, cuando los pobladores se relacionan cara a cara con sus representantes, lo que crea una inmediatez mayor entre los intereses de la población y el poder político (OVIEDO, 2017).

La Red de Mapa Verde de Holguín se encuentra en varias comunidades urbanas y rurales para enfrentar diversos problemas ambientales. Una de las comunidades en la que realizan un trabajo más sostenido es en el Consejo Popular Edecio Pérez, donde se han trabajado por más tiempo en la comunidad Oscar Lucero Moya, con la participación de diversos actores como la escuela del mismo nombre, la problemática socio-ambiental de la comunidad se trabaja a través de proyecto.

Está investigación problematiza la relación naturaleza sociedad en espacios comunitarios, en territorios donde los actores sociales muestran capacidades de transformación. Es así que coincidimos con Saquet (2017) al expresar que existe necesidad urgente de construir conocimientos contextualizados, con las singularidades y complejidades de las comunidades, la cartografía social ha sido utilizada como instrumento de participación comunitaria en el diálogo de saberes.

Se analiza el uso de la cartografía social como herramienta metodológica para la educación ambiental comunitaria, para ello se estudió el proceso de educación ambiental desarrollado en la comunidad Oscar Lucero Moya en la que se desarrolla el proyecto Mapa Verde con el proposito de promover la participación y el manejo local de problemas socioambientales. El objetivo de este estudio fue comprender la contribución de la cartografía social a la educación ambiental comunitaria a partir de presupuestos teóricos y prácticos. La cartografía social es una herramienta que posibilita la participación de 
diversos actores sociales en la solución de problemas ambientales.

La educación ambiental comunitaria ha de partir de la realidad del entorno local, de las aspiraciones e intereses de los sujetos sociales. En este proceso es importante la participación activa y crítica de las personas involucradas, también vincular lo aprendido con la vida cotidiana; esas condiciones permiten mejorar los saberes comunitarios y las habilidades para la transformación de realidades que afectan en lo cotidiano (ESPEJEL; FLORES, 2012).

\section{EDUCACIÓN AMBIENTAL COMUNITARIA E CARTOGRAFÍA SOCIAL}

La educación ambiental busca mejorar las relaciones entre las personas y su ambiente, a través del conocimiento, la promoción de estilos de comportamientos favorables al medio ambiente. Es tanto una educación para la adquisición de conocimientos y habilidades como una formación social y ética relacionada con el medio ambiente natural y construido, dirigida a sensibilizar y formar seres humanos más responsables (MARTÍNEZ, 2001). Como el foco del estudio es develar que ofrece la cartografía social a la educación ambiental comunitaria, consideramos pertinente iniciar el análisis con lo planteado por Trélles (2002).

La educación ambiental, en su enfoque comunitario es eminentemente participativa, busca compartir y no impartir; es un proceso interdisciplinar y complejo, es una educación que combina la teoría con la práctica, estimula el pensamiento crítico y la acción; busca la construcción colectiva del conocimiento, se orienta a la construcción de futuros alternativos (TRÉLLES, 2002).

La educación ambiental comunitaria puede ser realizada desde diferentes perspectivas metodológicas, la cartografía social según Herrera (2008) es una propuesta conceptual y metodológica que permite construir un conocimiento integral del territorio, desde la participación y el compromiso social.

La cartografía social al representar lo físico , no siempre se circunscribe a lo geográfico, también expresa formas de representar aquellos espacios no visibles que escapan de las representaciones gráficas y pertenecen a la cultura mediante la delimitación del territorio (BARRAGAN, 2016). La educación ambiental comunitaria precisa de la interacción con el contexto y la cartografía social ofrece la posibilidad de representar gráficamente las particularidades de los territorios.

La educación ambiental comunitaria, sugiere la consolidación de prácticas que promuevan principios éticos de auto responsabilidad y auto gestión, y por lo mismo, orientadas a deconstruir los valores de la racionalidad del modelo económico tradicional. Asumimos por naturaleza todo lo que existe y criticamos como esta se convirtió en recurso consumido y degradado en el proceso económico. La educación ambiental en las comunidades a de trabajar en la reconstrucción del concepto de naturaleza, es así que coincidimos con Leff (2016) al expresar que la racionalidad ambiental abre una interpretación descontructiva sobre la historia de desnaturalización de la naturaleza y un enfoque constructivista para resignificarla para su reapropiación cultural y reterritorialización.

Entre los beneficios de aprovechar la cartografía social en la educación ambiental comunitaria está que ofrece la oportunidad, para la representación del contexto mediante los mapas; contribuye a que los participantes representen los fenómenos sociales desde la multiplicidad de perspectivas o formas de analizar la realidad, lo que incluye la crítica a la manera de apropiarse de los recursos socioambientales.

La educación ambiental comunitaria otorga relevancia al contexto, lo que impone la necesidad de substituir los saberes y prácticas pedagógicas con una visión reduccionista del conocimiento, por propuestas ajustadas a saberes que conduzcan a los individuos a una visión multidimensional, en la que se analizan los fenómenos desde la complejidad. La complejidad discutida por Edgar Morin propone el desafío de otra forma de pensamiento y en consecuencia implicaciones importantes para la reflexión de la teoría y la práctica de la educación ambiental (SAHEB; RODRÍGUEZ, 2017).

La complejidad nos muestra las conexiones e interacciones que se producen entre todos los procesos en curso, es así que Morin (1996) expresa que esta se produce donde existe un enmarañamiento de acciones; sin embargo aprendemos a separar y simplificar. El pensamiento complejo es siempre local, situado en tiempo y momento; no es completo, sabe de antemano que siempre hay incerteza.

Los autores consideramos que la utilización de la cartografía social en acciones de educación ambiental comunitaria permite 
trabajar el pensamiento complejo, pues la cartografía social asume una visión sobre la realidad en términos epistemológicos y propone una metodología que plantea el análisis con el mapeo. Esta toma una mirada pos-crítica que se combina con elementos del pensamiento complejo de Edgar Morin (TELLO y GOROSTIAGA, 2009).

Entre las cuestiones centrales de la cartografía social está que permite visualizar e integrar diferentes saberes y perspectivas que contextualizan los problemas socio-ambientales y revelan como estos ocurren. En la construcción de los mapas las personas interactúan y priorizan los intereses colectivos ante lo individual, asumiendo una posición frente a la problemática. Utilizar la cartografía social para la educación ambiental significa pasar de prácticas pedagógicas a través de la escolarización e instrucción generalizada, a procesos de formación que fortalezcan la convivencia, la vida en comunidad, el desarrollo de sensibilidades y posiciones críticas relacionadas con la cultura y los ecosistemas.

Según Martínez (2010) entre los propósitos de la educación ambiental para el desarrollo sostenible se encuentran:

- Comprender la naturaleza compleja del ambiente resultante de la interacción de sus aspectos biológicos, físicos, sociales y culturales.

- Percibir la importancia del ambiente en las actividades de desarrollo económico, social y cultural.

- Mostrar las interdependencias económicas, políticas y ecológicas del mundo moderno en el que las decisiones y los comportamientos de todos pueden tener consecuencias.

- Comprender la relación entre los factores físicos, biológicos y socio-económicos del ambiente, así como su evolución y su modificación en el tiempo.

Utilizar la cartografía social en la educación ambiental comunitaria contribuye a los propósitos enunciados por Martínez (2010). También promueve una nueva relación de los actores sociales que participan en el proceso de educación ambiental, con la naturaleza, pues para poder realizar los mapas han de entrar en contacto con el conocimiento de los procesos ecológicos, territoriales, económicos, sociales y culturales, es decir, del análisis crítico de los problemas socio-ambientales locales y su relación con los modelos de gestión y las acciones humanas.
Los alcances de la cartografía participativa permite pensar en las posibilidades comunitarias más allá de las cartografías oficiales en las que las narraciones gráficas delimitan las subjetividades; esta opción alternativa, que algunos investigadores asocian con la investigación-acción participativa (IAP), cobra especial relevancia en los trabajos con comunidades y posee ciertas particularidades que la vinculan con la participación y la transformación (BARRAGÁN, 2016, p.251).

La cartografía social facilita las acciones de educación ambiental comunitaria, donde el contexto es relevante para la acción educativa. La educación ambiental en las comunidades ha de tener como eje central el proceso dialógico en el que las actividades sean amenas, sencillas y claras, logrando con ello que la gente hacia quien están dirigidas capte el mensaje y se despierte el interés por un mayor conocimiento de los procesos socio-ambientales, además se reconozca la diversidad lo que permite que se aplique y logren derechos fundamentales que son inherentes a las personas (DUQUE, QUINTERO \& DUQUE, 2014).

La educación ambiental comunitaria enfatiza en la relación teoría - práctica, teniendo en cuenta los aprendizajes a partir de proyectos, educación libre, talleres participativos, creación literaria, pintura, recorridos guiados, juegos y dinámicas. La educación ambiental comunitaria trabaja en torno a la recuperación y diálogo de saberes, además se dirige a la realización de prácticas vivenciales.

La cartografía social contribuye a una educación ambiental comunitaria activa, coincidimos con López (2019) al plantear que esta permite percibir las relaciones en el entorno y estudia las consecuencias de las interacciones en lo social y territorial, además puede ser considerada como herramienta de resolución de conflictos, donde las partes pueden transformar un problema en diversas soluciones creativas y dinámicas.

Es necesario que la educación ambiental que se realiza en las comunidades involucre a los actores sociales que conducen la vida en la comunidad e incluya métodos y prácticas educativas diseñadas para acentuar su enfoque interdisciplinar e intersubjetivo. Al combinar la cartografía social y la educación ambiental comunitaria se establecen puntos de conexión y diálogo, que promueven la construcción de 
bases más definidas en la materialización de comunidades sostenibles.

Una de las cuestiones centrales de la cartografía social es que permite visualizar y eventualmente integrar diferentes perspectivas que conceptualizan el fenómeno, y cómo estas interactúan o se interrelacionan. Por otra parte, aparece como un intento válido de responder al desafío de la post-modernidad de dar cuenta de diferentes perspectivas del conocimiento (TELLO \& GOROSTIAGA, 2009, p.166).

La educación ambiental comunitaria precisa de la integración de diferentes perspectivas, pues la crisis ambiental expresa los límites del crecimiento, así como la insustentabilidad de la racionalidad económica. Estos son los efectos de la historia del conocimiento occidental, de la universalidad de la verdad científica, de la hegemonía del pensamiento unidimensional, del productivismo, que reduce todas las cosas al valor monetario y al ordenamiento del mundo por las leyes del mercado (LEFF, 2016).

La educación ambiental comunitaria es capaz de contribuir al enfrentamiento de la crisis ambiental y hace del ambiente educativo espacios de participación, donde el aprendizaje se da en la construcción de un proceso de conocimientos, que promuevan acciones con la intencionalidad política de intervenir en la realidad y transformarla. Todo ese proceso en un ambiente educativo es propicio para una educación ambiental en su carácter crítico (GUIMARÃES, 2007).

La utilización de la cartografía social en la educación ambiental comunitaria va más allá de la representación gráfica, es así que coincidimos con Barragán (2016) al expresar que mediante la misma acontecen acciones educativas que implican vincular los participantes a transformar sus comunidades; en esas prácticas se fortalecen lazos de cooperación y se fortalece el aprendizaje entre pares. Así se resignifican las comprensiones de los diversos temas educativos, los cuales deben plasmarse cartográficamente a partir de la experiencia de los participantes.

Es así que la educación ambiental comunitaria se torna crítica y permite la participación de actores sociales en acciones dirigidas a transformar la realidad concreta, en la que se potencializan nuevas relaciones sociales con la naturaleza. No basta con apuntar los problemas y contradicciones ambientales, es necesario actuar. Para la educación ambiental crítica la práctica exige el conocimiento de las relaciones que se constituyen en la realidad, se conoce transformando (LOUREIRO; COSSIO, 2007).

La escuela al concebir la educación ambiental vinculada a la comunidad facilita la comprensión del mundo, no se limita a la acumulación de ideas y conocimientos pues somos seres de relaciones es a través de ellas que se adquiere la experiencia para enfrentar y adaptarnos a diversas situaciones. Es en el vínculo teoría - práctica que se comprende el impacto de nuestras acciones en el mundo. En otras palabras, si bien el conocimiento se desarrolla a través de ideas, categorías y conceptos, es en el encuentro con el mundo que desarrollamos una aprehensión profunda de la relación sociedad y naturaleza.

La educación ambiental comunitaria que utiliza la cartografía social, coloca el conocimiento de la comunidad en primer plano resaltando la percepción sobre su identidad y territorio, pues la creación de los mapas se da a partir de la selección de datos e información de la localidad. La aproximación a la comunidad muestra la subjetividad presente en el proceso, como los valores, creencias, intereses y voluntades de los sujetos involucrados en la construcción de los mapas (BARGAS; CARDOSO, 2015).

La educación ambiental comunitaria ha de tener en cuenta los aspectos educativos y sociales, porque las personas, los grupos y las comunidades son los afectados y beneficiarios de las transformaciones en el entorno físico. De igual forma, toda intervención socioeducativa tiene que contemplar los aspectos ambientales implicados, puesto que esta intervención siempre se desarrolla en un contexto socio-físico determinado. En este contexto, la educación ambiental comunitaria se convierte en elemento clave para la transformación del entorno, el desarrollo personal y social de las personas; en definitiva, lo que desde una concepción amplia podemos denominar desarrollo educativo/socio/ambiental sostenible (MUÑOZ; ESTEBAN, 2011).

$\mathrm{El}$ aprendizaje consciente de las relaciones entre los entornos y la vida no se da con la enseñanza descontextualizada del medio ambiente. Es solo a través del ejercicio educativo y reflexivo que los seres humanos comprendemos la naturaleza de la causalidad, es decir, la relación entre las acciones y sus repercusiones en los ecosistemas. Es en el universo de actuar donde se da la auténtica presencia de los individuos que mapean su 
realidad de lo contrario se quedaría en un simple diagnóstico (BARRAGÁN, 2016).

\section{Estrategia metodológica utilizada}

La investigación se realizó desde una perspectiva cualitativa, analizando la realidad desde la complejidad. La crisis ambiental es sobre todo un problema de conocimiento que lleva a repensar el ser de un mundo complejo. La complejidad ambiental irrumpe a partir de su negación por el pensamiento metafísico y por la alineación e incertidumbre de una racionalidad insustentable (LEFF, 2012).

En la investigación se analizó las contribuciones de la cartografía social a la educación ambiental comunitaria a través de un estudio de caso, para ello se seleccionó el proyecto Mapa Verde realizado en la comunidad Oscar Lucero Moya de la ciudad de Holguín. Según Álvarez San Fabián (2012) el estudio de caso posibilita el conocimiento de lo particular, de lo idiosincrásico, sin olvidar el contexto, se enfatiza en la contextualización del objeto de investigación, al entender que un estudio de caso es una investigación empírica dirigida a investigar un fenómeno contemporáneo dentro de su contexto real.

El proyecto Mapa Verde adoptó la cartografía social como metodología para materializar acciones de educación ambiental comunitaria, el mismo se ejecutó en tres etapas: la primera denominada "Diagnóstico Comunitario Socio-ambiental" que consistió en realizar actividades colectivas como talleres y excursiones para levantar de forma participativa los problemas socio-ambientales y luego analizarlos críticamente.

La segunda etapa "Simbología Comunitaria" en la que se realizó la representación gráfica de los problemas socio-ambientales, figurados simbólicamente en la construcción de varios mapas. Se crearon los grupos verdes quienes realizaron los recorridos y enseñaron la iconografía de Green Map System y estimularon la creación de simbología propia. La tercera etapa "Rehabilitación y Transformación Comunitaria" mediante la que se impulsaron acciones para cambiar la realidad a partir de significados y planificación colectiva.

Para estudiar la contribución de la cartografía social a la educación ambiental comunitaria en Oscar Lucero Moya se efectuaron entrevistas. Las entrevistas permiten escuchar los criterios de las personas que participaron en el proceso de educación ambiental; coincidimos con Duarte (2004) al expresar que las entrevistas nos permiten comprender la lógica de las relaciones que se establecen en el interior de los grupos sociales, de los cuales el entrevistado participa.

Fueron entrevistados 8 actores claves en la ejecución del proyecto de ellos dos profesores de la Universidad de Holguín quienes coordinaron el mismo, dos profesoras de la Escuela Oscar Lucero Moya, pues la escuela jugó un papel protagónico en la materialización de cada una de las etapas del proyecto Mapa Verde, el Presidente del Consejo Popular como la máxima representación del gobierno en el barrio con amplio conocimiento de la comunidad y por su labor en la articulación de actores a través del grupo comunitario que dirige, un líder comunitario por su contribución en la movilización de los pobladores, un representante de los Comité de Defensa de la Revolución (CDR) y una de la Federación de Mujeres Cubanas (FMC), estas son organizaciones con amplia membresía en la comunidad y participaron activamente en la ejecución del proyecto . Además se aplicó un cuestionario a 111 pobladores de la comunidad para conocer su criterio sobre la participación en las acciones promovidas por el proyecto.

La comunidad en la que se realizó el estudio es periurbana su nombre "Oscar Lucero Moya", esta pertenece al Consejo Popular "Edecio Pérez", ubicado en la ciudad de Holguín. Limita al norte con el Distrito Lenin y Purnio, al sur con Pedernales y la zona industrial, por el este con Harlem y por el oeste con Yareyal, se encuentra situada al centro y oeste del municipio, dividido por la carretera central.

Es una comunidad con 254 viviendas, en las que habitan los 1734 pobladores. El 57\% de sus habitantes es de procedencia rural y el $43 \%$ urbana, lo cual determina el carácter mixto de su población en cuanto al origen. Otro aspecto importante es que alrededor del $40 \%$ ha vivido en la comunidad por un período entre 30 y 40 años y solo el 33\% nació en ella. Existen problemas sociales como alcoholismo y desarraigo pues del $27 \%$ de los pobladores llegaron provisionalmente como albergados por haber perdido sus viviendas al paso del huracán George en 1998, lo que ocasionó conflictos al permanecer por seis años en esa condición provocando desarraigo y afectando el patrimonio edificado con valores históricos.

La comunidad posee valores patrimoniales y potencialidades como espacio físico ambiental pues está ubicada en la cuenca del río Matamoros, y este constituye un símbolo para sus pobladores; les brinda además un lugar de 
esparcimiento y aunque su nivel de contaminación no es favorable para el baño, de manera negligente, muchas personas lo utilizan con este fin. Está próxima también al área protegida "Dos Ríos", de altos valores de flora endémica local.

\section{RESULTADOS Y DISCUSIÓN}

\section{Educación ambiental \& cartografía social en Oscar Lucero Moya Holguín- Cuba}

El proyecto Mapa Verde es la forma en la que se aplica la cartografía social en el proceso de educación ambiental comunitaria desarrollado en Oscar Lucero Moya. Esta práctica permite comprender las contribuciones de la cartografía social al proceso de educación ambiental, pues la misma posibilita la construcción colectiva de conocimiento a través de la elaboración de mapas, donde se promueve la comunicación entre los participantes y se ponen en evidencia diferentes tipos de saberes que se mezclan para llegar a una imagen colectiva de la comunidad.

En entrevista realizada a los profesores de la Universidad de Holguín que coordinaron el proyecto Mapa Verde estos expresaron que en la educación ambiental comunitaria realizada trabajaron la formación de ambos grupos verdes, donde abordaron temáticas que favorecieron la concientización para el uso sustentable de los recursos socio-ambientales, comentaron que contribuyeron con una nueva cosmovisión del mundo dirigida a deconstruir los viejos paradigmas que separan al hombre y la naturaleza. Manifestaron también que trabajaron desde la interdisciplinaridad y consideraron las complejidades del entorno al mapear para que visibilicen los problemas socio-ambientales y condujeran acciones para la transformación.

$\mathrm{Al}$ analizar la formación realizada a los mapeadores se devela còmo la cartografía social permite comprender mejor la relación entre sociedad y naturaleza abordada a través de los problemas socio-ambientales del contexto. Los mapas pueden reflejar cualquier tema, lo que permite trabajar los temas socio-ambientales en la comunidad. A través de ellos se ponen al descubierto conflictos y problemas de la comunidad con el propósito de reconocerlos para poder cambiarlos. Los mapas pueden abarcar información diversa, según las necesidades e intereses de cada lugar (BRACERAS, 2012).

El saber ambiental construye su utopía a partir del potencial de lo real y de la realización de deseos que activa principios materiales y significaciones sociales para la construcción de una nueva realidad, en la que pueden movilizarse procesos para la realización de ciertos objetivos (LEFF, 2012). Mediante la educación ambiental en la comunidad se trabajaron las características socio-ambientales de la misma, la biodiversidad local, los riesgos socio-naturales y la prospectiva comunitaria.

La elaboración de mapas verdes en la comunidad Oscar Lucero Moya se realizó de forma participativa, la escuela junto a los pobladores identificaron los sitios relevantes de la misma, se ubicaron los lugares con conflictos socio-ambientales como el río Matamoro, el área protegida Dos Ríos y las áreas productivas de la cooperativa Atanagildo Cajigal. Como es una comunidad situada en los límites de la ciudad son evidentes los conflictos urbanos identificados como: la falta de servicios básicos de telefonía, transporte; y los rurales en relación al uso de los recursos naturales como el río, el área de preservación natural y las actividades agropecuarias.

Los conflictos en el río Matamoros son por la contaminación del agua pues al no existir sistemas de tratamiento las entidades y hogares descargan aguas residuales, lo que impide que los pobladores puedan utilizar el agua para el consumo y la recreación.

Otro de los conflictos identificados fue la acción antrópica en el área protegida Dos Ríos que está provocando la extinción de especies endémicas como el cactus enano (Escobaria cubensis). También diagnosticaron riesgos como el incremento de los períodos secos y los incendios forestales.

En entrevista con los profesores de la escuela ubicada en la comunidad estos expresaron que para elaborar los mapas verdes con las características de la comunidad se realizaron recorridos y talleres con la participación de pobladores y actores institucionales y gubernamentales. La figura 1 muestra uno de los mapas elaborados por los comunitarios. También diseñaron el mapa de la biodiversidad, otro con los valores patrimoniales y el mapa de los sueños donde colocaron las transformaciones planificas y las alcanzadas. 
Figura 1. Mapa de riesgo diseñado por pobladores de la comunidad Oscar Lucero.

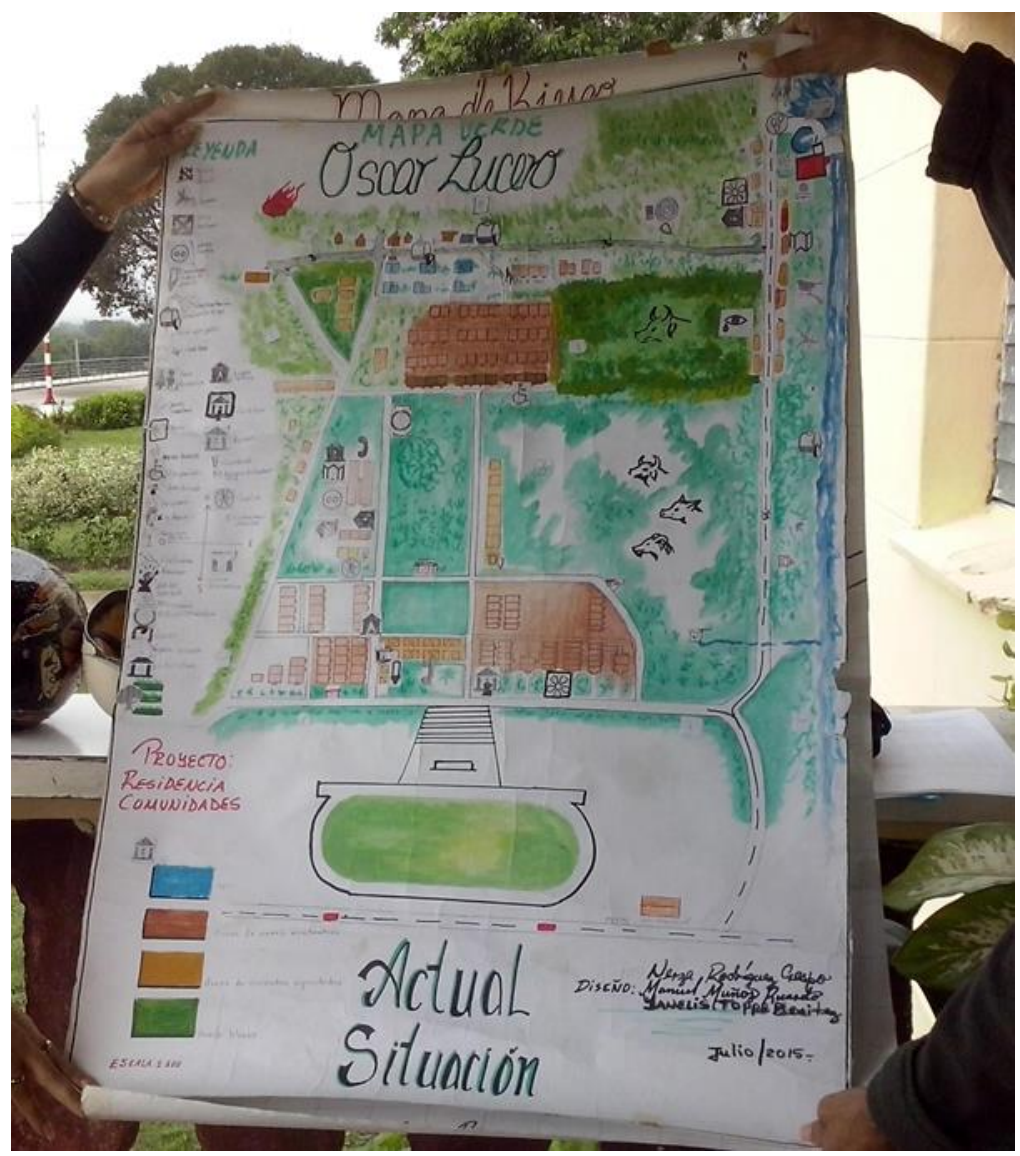

Fuente: Foto tomada por los autores

La cartografía social empleada levantó los conflictos socio-ambientales, contribuyó a fortalecer el sentido de pertenencia, a conocer los lugares más vulnerables ante inundaciones e incendios forestales de la comunidad Oscar Lucero Moya, además brindó información para la toma de decisiones, permitió el intercambio de ideas a través de la comunicación horizontal entre quienes participaron, asimismo esta acción fue el resultado de alianzas entre la escuela, grupo comunitario y el centro cultural. La realización de mapas contribuyó a la movilización y articulación entre los actores de la comunidad, lo que facilitó la participación en el proceso de educación ambiental comunitaria.

El proceso de confección de los mapas permite el involucramiento práctico, simbólico de quienes participan y se establecen interrelaciones. De esa forma el conocimiento de las comunidades locales se sitúa en primer plano, resaltando la percepción que tienen sobre su territorio. Se destaca además los valores y creencias de los sujetos que participan (BARGAS; CARDOSO, 2015).

La cartografía social contribuyó a la educación ambiental comunitaria en Oscar Lucero con prácticas educativas en las que se involucran varios actores como la Escuela, el Grupo Comunitario, organizaciones no gubernamentales como la Asociación de Combatientes de la revolución Cubana (ACRC), Federación de Mujeres de Cuba (FMC), Comité de Defensa de la Revolución (CDR), también organizaciones políticas como el Partido Comunista de Cuba (PCC), además instituciones como el Consultorio Médico, el Centro Cultural, la Cooperativa Agropecuaria "Atanagildo Cagigal", el Organopónico, entre otras, todas representadas en la figura 2. Sus acciones sirvieron para dar respuesta a necesidades colectivas y a la creación de alianzas en busca de un objetivo común, así mismo modificaron los conocimientos individuales y de los grupos sociales que participan, trabajan a partir de una realidad cotidiana y valorizan los saberes populares.

Coincidimos con Bozzano (2017) al plantear que los actores sociales contribuyen a comprender las relaciones sociales con el medio natural y construido, sus contradicciones, conflictos y relaciones de poder. Los actores sociales cargan consigo intereses cognitivos y materiales que representan a un colectivo, institución, organización política, económica o 
ciudadana; estos encarnan y significan los territorios.

La participación de diversos actores en el los procesos de educación ambiental comunitaria proporciona el intercambio de saberes y el empoderamiento de los sujetos que se involucran. Asímismo, permite la coordinación de tareas colectivas, en las que se implican intereses sociales e individuales. Estamos entendiendo como actores sociales los agentes institucionales, las organizaciones gubernamentales, no gubernamentales y políticas que interactuaron en las prácticas de educación ambiental comunitaria promovidas por el proyecto Mapa Verde.

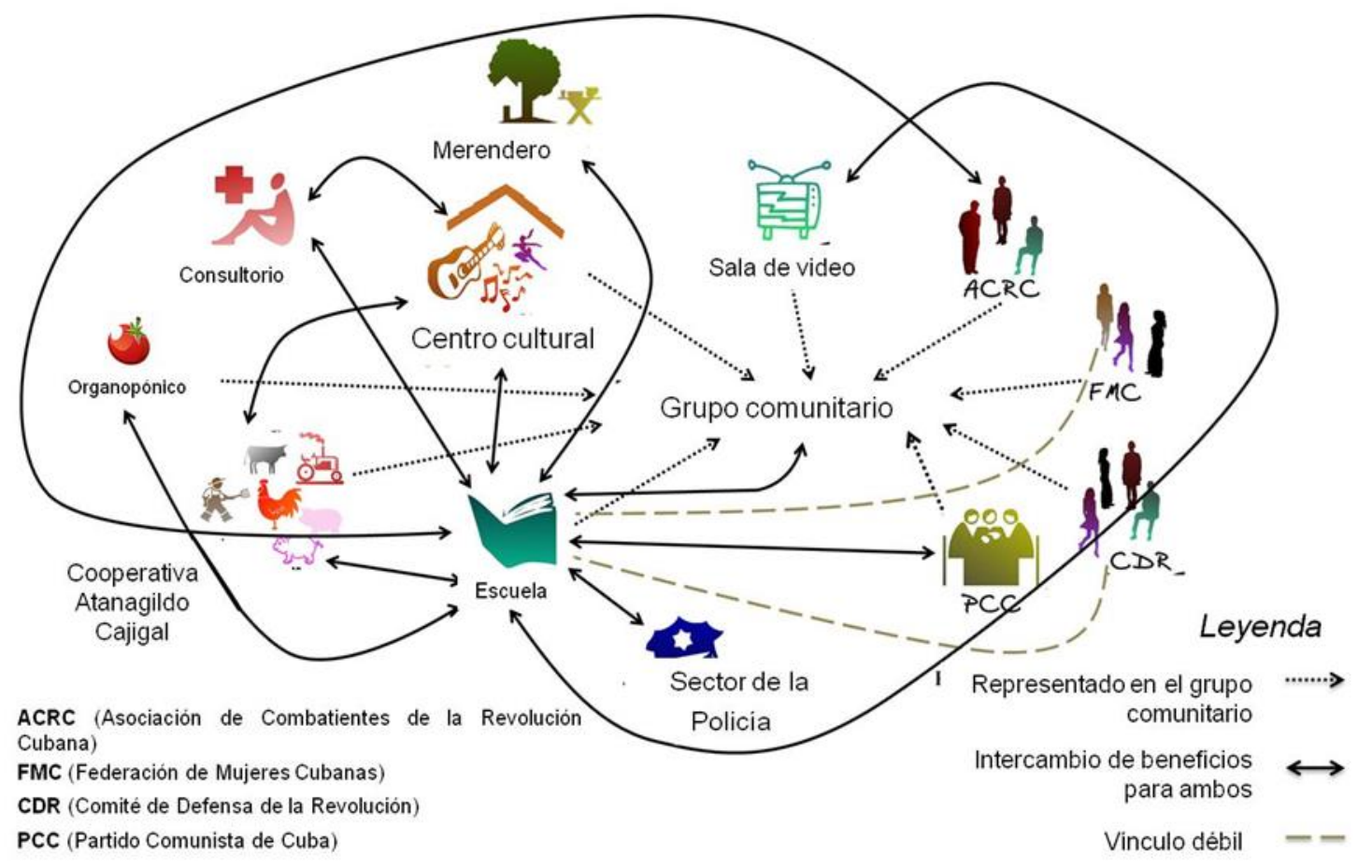

Fuente: Elaborado por los autores

Como está representado en la figura 2 la escuela en Cuba es considerada centro sociocultural de la comunidad, la escuela Oscar Lucero Moya se ha logrado articular fuertemente con actores institucionales $y$ organizaciones no gubernamentales que existen en la comunidad. Las acciones de educación ambiental comunitaria se realizan con la participación de varios actores sociales, considerados estos como sujetos activos y no objetos de prácticas externas.

La participación es significativa en los procesos de educación ambiental comunitaria, se puede ejercer mediante el análisis crítico de las necesidades y problemas, la evaluación de alternativas y el balance de los recursos disponible; cuando se participa en acciones comunitarias emerge el sentido de pertenecer. El sentimiento de pertenencia contiene el germen del compromiso con el todo en el cual se siente incluido, la toma de decisiones colectivas encarna la concreción de la participación real y constituye la vía para el ejercicio del protagonismo ciudadano, (REBELLATO, 2004).
Al preguntar a través del cuestionario a 111 pobladores de la comunidad Oscar Lucero Moya sobre su participación en las acciones de educación ambiental comunitarias promovidas por el proyecto Mapa Verde, el 78,38\% respondió que participan.

Aunque 78,38\% de los encuestados manifestó que participan en las actividades de educación ambiental comunitaria, en nuestro criterio aún es limitada la forma en que se involucran los pobladores en las acciones de educación ambiental, coincidimos con Conde (2009) al plantear que en Cuba es restringida la participación de la comunidad en los procesos de educación ambiental y casi nula en la evaluación de impacto y aún más escasa en la sistematización comunitaria, como proceso de reflexión crítica de una o varias experiencias.

En las entrevistas con el Presidente del Consejo Popular, un líder comunitario, un representante de los Comité de Defensa de la Revolución (CDR) y una de la Federación de Mujeres Cubanas (FMC) se les preguntó cómo 
se podía mejorar la participación en la comunidad, a continuación se relacionan algunos de los criterios colocados:

- Trabajando más en la comunidad pues las cosas mejoran con el fruto del trabajo.

- Ejecutando más actividades en el centro cultural.

- Diseñar actividades para los jóvenes y lograr mejor divulgación.

- A través del estímulo a los comunitarios y el trabajo educativo con ellos, para favorecer su cultura, puesto que el nivel educacional de algunos pobladores es bajo.

- Realizando más actividades donde se involucren a los jóvenes y demás vecinos de la comunidad.

- Organizando actividades culturales y recreativas.

Para Tréllez (2015) participar en acciones de educación ambiental comunitaria no es simplemente que las personas formen parte de algo, sino que se conviertan en expresión activa de procesos de transformación de sus territorios. El empleo de la cartografía social en Oscar Lucero Moya a través del proyecto Mapa Verde permitió materializar otras acciones educativas importantes como la creación del bosque martiano, se realizó en la escuela Oscar Lucero Moya y permitió sembrar en el patio las especies de árboles descritas por José Martí (Héroe Nacional de Cuba) en su diario de campaña.

Esta actividad además de ser significativa para la conservación de la biodiversidad pues se plantaron 23 especies de árboles, tiene un importante contenido patriótico e histórico al resaltar los valores del héroe. De esa manera abordaron problemas del presente a partir de la memoria histórica recogida en el diario, en el que se describen 32 especies arbóreas pertenecientes a 34 géneros de 20 familias agroforestales, algunas de ellas en peligro de extinción y otras extintas.

También se realizaron transformaciones en el espacio físico de la comunidad pues se rehabilitó las edificaciones con valor patrimonial, esas edificaciones tienen un significado histórico, porque primero fueron cuartel militar de la dictadura de Batista y al triunfar la revolución cubana en 1959 fue convertido en escuela, al construirse nuevas edificaciones para escuelas, el gobierno las utilizó como albergues para viviendas a personas que perdieron sus casas al paso del huracán George en 1998, después de seis años los albergues se convirtieron en viviendas y las familias comenzaron a vivir de forma permanente en Oscar Lucero Moya.

La cartografía social realizada mediante el proyecto Mapa Verde consideró los valores patrimoniales que posee la comunidad, como las edificaciones que fueron primero cuartel militar, luego escuelas y después viviendas, estas constituyen un elemento de identidad importante, que favoreció la transformación comunitaria. Por lo cual se puede afirmar que el patrimonio se convierte en un lugar de complicidad social, donde se expresa la solidaridad que une a quienes comparten los mismos valores culturales (GARCÍA, 2009).

En las entrevistas el presidente del Consejo Popular, las dos profesoras de la escuela y los representantes de los CDR y FMC coincidieron en plantear que las acciones efectuadas en la comunidad han permitido mejorar el sentido de pertenencia y a reconocer los valores históricos y patrimoniales. Lo que nos lleva a pensar que una de las contribuciones de la cartografía social a la educación ambiental comunitaria en Oscar Lucero Moya fue que permitió que los pobladores involucrados visibilizaran los valores arquitectónicos e históricos de su comunidad, lo que puede atenuar el problema de desarraigo de quienes habitaban de forma provisional y pasaron a residir permanentemente.

Las acciones de educación ambiental comunitaria no solo fueron dirigidas a rehabilitar el medio construido, también se dirigió a las áreas naturales como la reserva florística Dos Ríos ubicada en las proximidades de la comunidad. Lo que fue aprovechado para realizar actividades educativas, como la identificación de especies, estudio de los valores florísticos más significativos del área y la recuperación de especies nativas y endémicas, Lo que posibilitó no solo vivenciar la naturaleza, también generó aprendizaje colaborativo en las excursiones efectuadas.

Las excursiones aportaron información para elaborar mapas con las especies de la flora en peligro en la reserva florística Dos Ríos, lo que permitió intercambiar saberes sobre la biodiversidad del lugar; concordamos con López (2019) cuando plantea que la cartografía social es en esencia educativa porque permite la construcción colectiva de conocimientos, que se concretan en la construcción de los mapas que ponen en juego diferentes tipos de saberes.

La educación ambiental comunitaria desplegada en la comunidad Oscar Lucero 
Moya mediante el proyecto Mapa Verde se ajustó al contexto, estableciendo diálogo ente los participantes, considerando la complejidad e interdisciplinaridad de la temática ambiental. Los temas analizados surgieron de la realidad local, aprender haciendo fue uno de los principios aplicados, las habilidades se desarrollaron combinando actividades teóricas con acciones prácticas.

Consideramos las acciones de educación ambiental comunitaria efectuadas en Oscar Lucero Moya como transformadoras, es así que coincidimos con Ferreira (2013) al expresar que la educación ambiental sola no conseguirá transformar la sociedad, aunque puede ser el punto de partida para incentivar reflexiones y quehaceres que contribuyan a disminuir los daños ambientales. El espacio creativo y motivador en el proceso de educación ambiental en las comunidades favorece nuevas ideas que contribuyen a la construcción de sociedades sustentables.

En Oscar Lucero Moya el proceso de educación ambiental contó con el apoyo de los líderes comunitarios, el liderazgo no es algo que los dirigentes simplemente llevan a cabo, está centrado en las interacciones. Hace dos décadas este comenzó a verse más como propio de una comunidad que de un individuo, pues es asumido por diversas personas en diversos contextos (ANDRADE, 2013). Para Paulo Freire, el liderazgo ha de prosperar paralelamente al crecimiento del todo, basándose en una percepción social nueva que construyen en conjunto, si no ocurre así, es sustituido por los líderes que emergen (FREIRE, 1997).

Los líderes comunitarios de Oscar Lucero Moya fueron intermediarios entre los agentes externos como los profesores de la Universidad de Holguín y la comunidad, convirtiéndose de esta forma en actores claves, estos representan las demandas de la comunidad y es a través de ellos que se organiza. Cuando un líder deja de representar los intereses colectivos, simplemente la comunidad deja de legitimarlo; esta no es una posición estática es un proceso de expansión y contracción constante de líderes cuya visibilidad depende de las necesidades de la comunidad (ANDRADE, 2013).

Los líderes comunitarios de Oscar Lucero Moya fueron actores decisivos pues condujeron las acciones colectivas, que según Barragán \& Amador (2014) es lo más importante de cualquier cartografía social, pues los componentes políticos, culturales e interpersonales hacen de la reconstrucción virtual de la realidad una manera legítima de construir mundos posibles.

\section{CONSIDERACIONES FINALES}

El estudio del proyecto Mapa Verde en la comunidad Oscar Lucero Moya mostró las contribuciones de la cartografía social a la educación ambiental comunitaria, pues:

- Mediante esta los pobladores levantaron los problemas socio-ambientales en reflexión conjunta, en diálogo de saberes se compartió sobre biodiversidad, gestión del riesgo y sustentabilidad. Materializaron acciones dirigidas a la reforestación, protección de la flora autóctona, identificación de vulnerabilidades por inundaciones e incendios forestales y el rescate de edificaciones con valor patrimonial;

- Se estimuló la participación de actores sociales en acciones dirigidas a transformar la realidad socio-ambiental de la comunidad. Los líderes ocuparon una posición relevante, lo que facilitó la coordinación de tareas colectivas, en las que se implicaron intereses sociales e individuales;

- La escuela se convirtió en el centro sociocultural más importante de la comunidad, al lograr articularse fuertemente con otros actores existentes en el territorio y con la Universidad de Holguín, también con organizaciones no gubernamentales como los CDR y la FMC. Lo que permitió actividades interinstitucionales e interdisciplinares;

- Se potencializó nuevas relaciones sociales con la naturaleza, en la práctica se fortalecieron lazos de cooperación y se construyeron nuevos saberes que resignificaron el contexto. Además se asumió una nueva cosmosvisión desde un enfoque de sistema complejo, que favoreció el análisis de los conflictos socio-ambientales develados como problemas.

\section{REFERENCIAS}

ÁLVAREZ, C.; SAN FABIAN, J. L. La elección del estudio de caso en investigación educativa. Gaceta de Antropología, v.28, n.1, p.1-12, 
2012. http://hdl.handle.net/10481/20644 .Acceso: Marzo 15, 2018.

ANDRADE, R. El liderazgo comunitario y su importancia en la intervención comunitaria. Psicologia América Latina, n.25, p. 57-76, 2013.

http://pepsic.bvsalud.org/scielo.php?script=sci_a bstract\&pid=S1870 -

350X2013000200005\&lng=pt\&nrm=iso\&tlng=es . Acceso:Febrero 22, 2018.

BARGAS, J.; CARDOSO, L. F. Cartografia social e organização política das comunidades remanescentes de quilombos de Salvaterra, Marajó, Pará, Brasil. Bol. Mus. Para. Emílio Goeldi. Cienc. Hum, Belém, v. 10, n. 2, p. 469488, 2015. https://doi.org/10.1590/198181222015000200013

BARRAGAN, D. F. Cartografía social pedagógica: entre teoría y metodología. Revista Colombiana de Educación, n. 70, p.247-285, 2016.

https://doi.org/10.17227/01203916.70rce247.285

D. F, AMADOR, J. C. La

cartografía social- pedagógica: Una oportunidad para producir conocimientos y repensar la educación. Itinerario Educativo, n.64, p.127141 2014.

https://doi.org/10.21500/01212753.1422

BOZZANO, H. Territorios posibles. Procesos, lugares y actores. Buenos Aires: Lumiere, 3ra edición, 2017.

BRACERAS, I. Cartografía participativa: herramienta de empoderamiento y participación por el derecho al territorio. Universidad del País Vasco, 2012.

CONDE, E. Enfoques pedagógicos para la educación ambiental, 2009. Disponible en: https://www.gestiopolis.com/enfoques-

pedagogicos-para-la-educacion-ambiental/. Acceso: Julio 5, 2018.

DUARTE, R. Entrevistas em pesquisas qualitativas. Educar, Curitiba, n.24, p.213-225, 2004. https://doi.org/10.1590/0104-4060.357

DUQUE, S. P; QUINTERO, M. L.; DUQUE, M. La educación ambiental en comunidades rurales y la popularización del derecho a la conservación del entorno natural: el caso de la comunidad de pescadores en la ciénaga de Ayapel (Colombia). Revista Luna Azul, n.39, p.6-24, 2014. https://doi.org/10.17151/luaz.2014.39.2

ESPEJEL, A.; FLORES, A. Educación ambiental escolar y comunitaria en el nivel medio superior. Revista Mexicana de Investigación Educativa, v.17, n.55, p.1173-1199, 2012. http://www.scielo.org.mx/pdf/rmie/v17n55/v17n5 5a8.pdf . Acceso: Febrero 23, 2018.

FERREIRA, C. E. O meio ambiente na prática de escolas públicas da rede estadual de São Paulo: intenções e possibilidades. Ambiente \& Educação, v.18, n.1, p.185-209, 2013. https://periodicos.furg.br/ambeduc/article/view/3
100/2408. Acceso: Enero31, 2018

FREIRE, P. Educación como práctica de la libertad. México D.F: Siglo Veintiuno Editores, S.A, 1997.

GARCÍA, Z. ¿Cómo acercar los bienes patrimoniales a los ciudadanos? Educación Patrimonial, un campo emergente en la gestión del patrimonio cultural, Pasos Revista de Turismo y Parimonio Cultural, vol.7, n.2, pp.271-280, 2009 . https://doi.org/10.25145/j.pasos.2009.07.019

GUIMARÃES, M. Educação Ambiental: participação para além dos muros da escola. In DE MELHO, S; TRAJBER, R (Coord). Vamos a cuidar do Brasil. Conceitos e práticas em educação ambiental na escola. Brasília: UNESCO, Ministério da Educação, Ministério do Meio Ambiente, 2007, p.85-94.

HERRERA, J. Cartografía Social. España: Universidad de la Laguna, 2008. Disponible en: https://juanherrera.files.wordpress.com/2008/01/ cartografia-social.pdf. Acceso: Enero 25, 2018.

$\mathrm{LEFF}$, E. A aposta pela vida. Imaginação sociológica e imaginários sociais nos territórios ambientais do sul. Petrópolis: Vocês, 2016

Aventuras da epistemologia ambiental. Da articulação das ciências ao diálogo de saberes. São Paulo: Cortez, 2012.

LOPEZ, C. E. La cartografia social como herramienta educativa. Revista Scientific, v.3, n.10, p.233-246, 2019. https://doi.org/10.29394/Scientific.issn.25422987.2018.3.10.12.232-247

LOUREIRO, C. F.; COSSIO, M. Um olhar sobre a educação ambiental nas escolas: considerações inicias sobre os resultados do projeto "O que fazem as escolas que dizem que fazem educação ambiental?" ". In DE MELHO, S; TRAJBER, R (Coord). Vamos a cuidar do Brasil. Conceitos e práticas em educação ambiental na escola. Brasília: UNESCO, Ministério da Educação, Ministério do Meio Ambiente, 2007, p.57-64.

MARTÍNEZ, J. Gestión ambiental y participación una aproximación inicial. Quito: Red Cántaro, 2001.

MARTÍNEZ, R. La importancia de la educación ambiental ante la problemática actual. Revista Electrónica Educare, v. XIV, n. 1, p. 97-111, 2010. https://doi.org/10.15359/ree.14-1.9

MORIN, E. Epistemologia da complexidade. In: SCHNITMAN, Dora Fried (Org.). Novos Paradigmas, culura e sujetividade. Porto Alegre: Artes Medicas, p.275-289, 1996.

MUÑOZ, L.; ESTEBAN, M. Desde la educación social a la educación ambiental. Hacía una intervención educativa socioambiental. Revista de Humanidades, n. 18, 2011. https://doi.org/10.5944/rdh.18.2011.12885

OVIEDO, V. La educación popular ambiental y la participación social en la gestión ambiental 
comunitaria: experiencias sociales en dos barrios de la Habana. Revista Cubana de Ciencias Sociales, n.46, p.125-144, 2017.

POGGI, Z. Nueva Cartografía Social. Cuadernos del CENDES, vol. 30, núm. 83, pp. 135-139, 2013.

http://ve.scielo.org/pdf/cdc/v30n83/art09.pdf.

Acceso: Marzo 15, 2018.

REBELLATO, J. L. La participación como territorio de contradicciones éticas. In ROMERO, M. I; NORA, C. Concepción y metodología de la educación popular. Selección de Lecturas Tomo I. La Habana: Caminos, p.299-336, 2004.

SAHEB, D.; RODRIGUEZ, D. A contribuição da complexidade de Morin para as pesquisas em educação ambiental. Revista eletrônica do Mestrado em Educação Ambiental. Edição especial XVI Encontro Paranaense de Educação Ambiental, p.191-207, 2017. https://doi.org/10.14295/remea.v0i0.7139

SAQUET, M. Consciência de classe e de lugar, práxis e desenvolvimento territorial. Rio de Janeiro: Consequência, 2017.

SUAVÉ, L. Una cartografía de corrientes en educación ambiental. In Sato, Michele, Carvalho, Isabel (Orgs). A pesquisa em educação ambiental: cartografias de uma identidade narrativa em formação. Porto Alegre: Artmed, 2004.

TELLO, C.; GOROSTIAGA, J. El enfoque de cartografia social para el análises de debates sobre políticas educativas. Práxis Educativa, Ponta Grossa, v.4, n.2, p.159-168, 2009. https://doi.org/10.5212/PraxEduc.v.4i2.159168

TRÉLLES, E. La educación ambiental comunitaria y la retrospectiva: Una alianza de futuro. Tópicos en educación ambiental, v.4, n.10, p.7-21, 2002 . <http://anea.org.mx/Topicos/T\%2010/Pagina\%20 07-21.pdf. Acceso: Enero 25, 2018.

\section{Educación ambiental} comunitaria en América Latina. PNUMA, 2015.

VÉLEZ, I.; RÁTIVA, S.; VARELA, D. Cartografía social como metodología participativa y colaborativa de investigación en el territorio afro- descendiente de la cuenca alta del río Cauca. Cuad. Geografía. Rev. Colombiana de Geografía, v.21, n. 2, p. 59 - 73, 2012. https://doi.org/10.15446/rcdg.v21n2.25774 . 